\title{
A comparison of plasma and prostate lycopene in response to typical servings of tomato soup, sauce or juice in men before prostatectomy
}

\author{
Elizabeth M. Grainger ${ }^{1,2} \dagger$, Craig W. Hadley ${ }^{3} \dagger \neq$, Nancy E. Moran ${ }^{2}$, Kenneth M. Riedl ${ }^{2,3}$, \\ Michael C. Gong ${ }^{4}$ \, Kamal Pohar ${ }^{4}$, Steven J. Schwartz ${ }^{2,3}$ and Steven K. Clinton ${ }^{1,2 *}$ \\ ${ }^{1}$ Division of Oncology, Department of Internal Medicine, College of Medicine, The Obio State University, \\ A456 Starling Loving Hall, 320 West 10th Avenue, Columbus, OH 43210, USA \\ ${ }^{2}$ The Obio State University Comprehensive Cancer Center, College of Medicine, The Obio State University, \\ Columbus, OH 43210, USA \\ ${ }^{3}$ Department of Food Science and Technology, College of Food, Agriculture, and Environmental Sciences, \\ The Obio State University, Columbus, OH 43210, USA \\ ${ }^{4}$ Department of Urology, College of Medicine, The Obio State University, Columbus, OH 43210, USA \\ (Submitted 18 February 2015 - Final revision received 15 May 2015 - Accepted 27 May 2015 - First published online 23 July 2015)
}

\begin{abstract}
Tomato product consumption and estimated lycopene intake are hypothesised to reduce the risk of prostate cancer. To define the impact of typical servings of commercially available tomato products on resultant plasma and prostate lycopene concentrations, men scheduled to undergo prostatectomy ( $n$ 33) were randomised either to a lycopene-restricted control group ( $<5 \mathrm{mg}$ lycopene/d) or to a tomato soup (2-2 $\frac{3}{4}$ cups prepared/d), tomato sauce $(142-198 \mathrm{~g} / \mathrm{d}$ or $5-7$ ounces/d) or vegetable juice $(325-488 \mathrm{ml} / \mathrm{d}$ or $11-16.5 \mathrm{fluid}$ ounces/d) intervention providing $25-35 \mathrm{mg}$ lycopene/d. Plasma and prostate carotenoid concentrations were measured by HPLC. Tomato soup, sauce and juice consumption significantly increased plasma lycopene concentration from 0.68 (SEM 0.1) to 1.13 (sEM 0.09) $\mu \mathrm{mol} / 1$ (66\%), $0 \cdot 48$ (SEM 0.09$)$ to $0.82(\operatorname{sem~} 0.12) \mu \mathrm{mol} / 1(71 \%)$ and 0.49 (SEM 0.12) to 0.78 (SEM $0 \cdot 1) \mu \mathrm{mol} / 1$ (59\%), respectively, while the controls consuming the lycopene-restricted diet showed a decline in plasma lycopene concentration from 0.55 (SEM $0 \cdot 60$ ) to 0.42 (SEM 0.07$) \mu \mathrm{mol} / 1(-24 \%$ ). The end-of-study prostate lycopene concentration was $0 \cdot 16$ ( tomato soup $(P=0.001)$, sauce $(P=0.001)$ and juice $(P=0.165)$ consumers, respectively. Prostate lycopene concentration was moderately correlated with post-intervention plasma lycopene concentrations $(r 0.60, P=0.001)$, indicating that additional factors have an impact on tissue concentrations. While the primary geometric lycopene isomer in tomato products was all-trans (80-90\%), plasma and prostate isomers were 47 and $80 \%$ cis, respectively, demonstrating a shift towards cis accumulation. Consumption of typical servings of processed tomato products results in differing plasma and prostate lycopene concentrations. Factors including meal composition and genetics deserve further evaluation to determine their impacts on lycopene absorption and biodistribution.
\end{abstract}

\section{Key words: Lycopene isomers: Lycopene: Prostate cancer: Prostatectomy: Tomatoes}

A series of evaluations of the Health Professionals Follow-Up Study, a large prospective cohort study of men initiated in 1984, have consistently reported that more frequent tomato product intake and greater estimated lycopene exposure is associated with a reduced risk of advanced or lethal prostate cancer $^{(1-3)}$. The Health Professionals Follow-Up Study has the advantage of conducting multiple evaluations of dietary intake over decades and a careful pathological review, allowing investigators to specifically examine prostate cancer-specific death and cancers of varying grade and stage $^{(1-3)}$. Yet, many epidemiological studies have not detected a relationship between tomato intake and the risk of prostate cancer ${ }^{(4,5)}$, which is not surprising given the complexity of defining biologically significant prostate cancer, particularly in prostate-specific antigen (PSA)-screened populations $^{(6)}$. Tomato components exhibit bioactivity in

\footnotetext{
Abbreviation: PSA, prostate-specific antigen.

*Corresponding author: Dr S. K. Clinton, fax +1 614293 7525, email steven.clinton@osumc.edu

† Both authors contributed equally to this work.

‡C. W. H. is currently affiliated with Mead-Johnson Nutrition, Glenview, IL, USA

§M. C. G. is currently affiliated with the Cleveland Clinic, Cleveland, OH, USA.
} 
rodent models of prostate carcinogenesis, reducing severity and incidence ${ }^{(7-10)}$. The tomato bioactive component lycopene is the predominant carotenoid found in the prostate tissue of American men (average concentration approximately $0.5 \mathrm{nmol} / \mathrm{g}$; range $0-2.58 \mathrm{nmol} / \mathrm{g})^{(11-14)}$, and has been hypothesised to have an impact on prostate carcinogenesis through its ability to quench reactive oxygen ${ }^{(15-18)}$, alter growth factor and androgen signalling ${ }^{(19-22)}$, inhibit cell invasion and migration ${ }^{(23)}$, and enhance apoptosis ${ }^{(9,17,19,24-27)}$. Thus, studies have supported a hypothesis that dietary tomato or lycopene intake may inhibit prostate carcinogenesis, though a causal relationship is not established.

The dynamic connection between intake of lycopenecontaining foods and lycopene concentrations in the blood and prostate tissue has been examined in a limited number of studies, and quantitative relationships remain speculative. A nuanced understanding of these relationships may improve the interpretation of epidemiological studies and help investigators design clinical intervention studies to better assess causality. In epidemiological cohorts, the relationship between estimated intake (based upon FFQ) and blood concentrations of lycopene can be described as modest, with correlation coefficients ranging from $0 \cdot 21^{(28,29)}$ to $0 \cdot 28^{(30,31)}$. From epidemiology alone, it is unclear whether these low correlations are due to imprecise intake data, including the variability of lycopene content in foods from the National Nutrient Database for Standard Reference ${ }^{(32)}$, differences in food processing and cooking that have an impact on absorption ${ }^{(33)}$, and/or genetic and biological variables that influence biodistribution.

Only a few human intervention studies have examined prostate lycopene concentrations following a controlled dietary or dietary supplement intervention. Daily consumption of a tomato sauce-based pasta meal (30 mg lycopene/d) for 3 weeks increased prostate lycopene 3-fold (from 0.37 to $1.08 \mathrm{nmol} / \mathrm{g}$ tissue), in comparison with baseline biopsy samples in men with stage $\mathrm{T} 1$ or $\mathrm{T} 2$ prostate cancer undergoing prostatectomy ${ }^{(34)}$. Similarly, when men were provided with $30 \mathrm{mg}$ lycopene/d for $21 \mathrm{~d}$ as a tomato sauce-based pasta meal, the reported prostate lycopene concentration was $0.82(\operatorname{sem} 0 \cdot 12) \mathrm{nmol} / \mathrm{g}$ tissue ${ }^{(13,35)}$. Alternatively, while provision of a daily tomato oleoresin supplement containing $30 \mathrm{mg}$ lycopene for $21 \mathrm{~d}$ did lead to significantly higher final prostate lycopene concentrations compared with controls $(0.59$ (SEM 0.47$) \quad v$. $0.45(\operatorname{sem} 0.53) \mathrm{nmol} / \mathrm{g}$, respectively, $P=0.005)^{(35)}$, the modest impact appears to be lower than that of food products, suggesting food-based lycopene may be more bioavailable than supplemental lycopene. These studies indicate that short-term dietary tomato interventions have a significant impact on prostate lycopene concentration, though quantitative relationships and potential confounding factors are not well understood.

In an earlier study, we reported that tomato soup, sauce and juice products each resulted in different plasma lycopene concentrations in healthy adults ${ }^{(36)}$. However, the impact of specific tomato food products on prostate lycopene concentrations is unknown and is the focus of the present study. The information provided may guide our future efforts in the rational selection of commercial food products and/or the design of novel food products for human intervention studies to modify the risk of prostate cancer. Furthermore, understanding the relative effects of different processed tomato products on plasma and prostate lycopene concentrations will enhance our ability to interpret epidemiological studies. In addition, should a therapeutic range of prostate lycopene concentrations be defined, the question remains as to the utility of using plasma lycopene concentrations as a biomarker of prostate lycopene exposure.

Processed tomato products, including soup, sauce and juice, are popular, affordable and frequently consumed by Americans. Per capita, Americans consume $65 \mathrm{lbs}(29 \cdot 5 \mathrm{~kg})$ of processed tomato products, including, sauces, soups and juices, and $21 \mathrm{lbs}(9.5 \mathrm{~kg})$ of fresh tomatoes each year ${ }^{(37)}$. Tomatoes are a source of other carotenoids in addition to lycopene, including provitamin A $\beta$-carotene ${ }^{(12)}$. In the present study, we sought to (1) determine the impact of daily consumption of three tomato food products (sauce, juice and soup) in typically consumed serving sizes, compared with a controlled lycopene diet, on prostate and plasma lycopene concentrations in men with prostate cancer; (2) determine the correlation between plasma and prostate lycopene concentrations after the dietary intervention; and (3) describe lycopene isomer distribution in the plasma and prostate.

\section{Experimental methods}

\section{Subjects}

Study participants ( $n$ 33) had biopsy-proven, clinically localised adenocarcinoma of the prostate ( $n$ 31), severe benign prostatic hyperplasia ( $n$ ) or invasive cancer of the bladder ( $n$ 1), based on standard pre-surgical staging studies. Subjects chose to undergo a radical prostatectomy or a cystoprostatectomy for the treatment of their disease after the medical team had presented all possible treatment options. Men were excluded if they were currently taking specific dietary supplements containing lycopene or any herbal or botanical supplements marketed to influence prostate health. Additionally, men were excluded if they had a history of digestive or malabsorptive disorders, metabolic enzyme deficiencies, or other disorders requiring special dietary modifications (controlled type 2 diabetes mellitus was allowed). Men were recruited at the Ohio State University Medical Center, and research was conducted according to the guidelines of the Declaration of Helsinki. All procedures involving human subjects/patients were approved by the Ohio State University Biomedical Sciences Institutional Review Board and the Comprehensive Cancer Center Clinical Scientific Review Committee (Columbus, Ohio). Written informed consent was obtained from all patients. The trial was registered at ClinicalTrials.gov as NCT 02292524.

\section{Study design and intervention}

Subjects were instructed to complete a tomato-free washout diet for a minimum of 1 week before baseline blood sampling. 
Men were then randomised to one of four dietary interventions: a controlled lycopene diet $(\leq 5 \mathrm{mg} / \mathrm{d}$ from foods); daily spaghetti sauce (Prego ${ }^{\circledR}, 142-198 \mathrm{~g} / \mathrm{d}$ or $5-7$ ounces/d, providing $24-35 \mathrm{mg}$ lycopene/d); daily vegetable juice (V8 ${ }^{\circledR}$, $325-488 \mathrm{ml}$ or $11-16.5$ fluid ounces/d, providing $24-35 \mathrm{mg}$ lycopene/d); or daily tomato soup (Campbell's ${ }^{\circledR}$ Tomato Soup, $473-651 \mathrm{ml}$ or $2-2 \frac{3}{4} \mathrm{cups} / \mathrm{d}$, providing $24-33 \mathrm{mg}$ lycopene/d). All products were provided by the Campbell Soup Company. The interventions were to be followed until the scheduled surgery date, with a minimum $14 \mathrm{~d}$ intervention time required for study inclusion. The targeted intake was defined by typically consumed serving sizes in order to ensure participant compliance. The goal was to provide three commonly consumed tomato products in typical serving sizes for the delivery of $25-35 \mathrm{mg}$ lycopene/d in the context of a usual diet but devoid of other sources of tomato products. The amount of $\beta$-carotene provided daily was $0.44-0.62 \mathrm{mg}$ from the sauce, $0.98-1.47 \mathrm{mg}$ from the juice and $1.20-1.65 \mathrm{mg}$ from the soup, while other major carotenoids ( $\alpha$-carotene, lutein, zeaxanthin, $\alpha$-cryptoxanthin and $\beta$-cryptoxanthin) were not detectable. The analysis of lycopene isomers in tomato products by HPLC was described previously using a YMC C30 analytical column (Waters) and electrochemical detection ${ }^{(36)}$, and typical product $\beta$-carotene and other carotenoid concentrations were determined by HPLC equipped with a YMC C30 column and photodiode array detection as described previously ${ }^{(38)}$.

\section{Compliance and safety assessment}

Participants kept daily logs documenting consumption of the intervention product and compliance with the tomatorestricted diet from enrolment to the day of surgery. A previously described worksheet was used for subjects to track lycopene intake ${ }^{(39)}$. During the washout period, participants were instructed to avoid all food sources of tomatoes and to document unintentional consumption. Following the washout period, the control group was educated regarding a diet providing $\leq 5 \mathrm{mg}$ lycopene/d by using a list of permissible tomato foods and the corresponding serving sizes. Men in the tomato product intervention groups continued the tomato-free baseline diet for the remainder of the study and documented all deviations. They also used the daily log to document consumption and provide details about the volume of the tomato intervention product consumed. Subjects were queried by telephone about their tolerance of the study product several times during the intervention. Toxicity was evaluated using the National Cancer Institute Common Terminology Criteria for Adverse Events version 3.0

\section{Blood and tissue collection}

Blood samples were collected after a 1-week washout and on the day of surgery. Blood was collected for both standardof-care pre-surgical analyses (including blood chemistry, complete blood count and PSA) and study-specific analyses (including plasma carotenoid and vitamin A concentrations). Fresh prostate samples were obtained at the time of prostatectomy or cystoprostatectomy. Samples were collected, and all subsequent analyses were performed under subdued light to minimise carotenoid degradation and isomerisation.

\section{Biochemical analysis}

Plasma carotenoid, retinol and retinyl palmitate analysis. Venous blood was collected in $10 \mathrm{ml} \mathrm{K}_{3}$ EDTA tubes (Becton Dickinson) and centrifuged at $4^{\circ} \mathrm{C}$ and $1500 \mathrm{~g}$ for $20 \mathrm{~min}$. Plasma was removed and stored under $\mathrm{N}_{2}$ at $-80^{\circ} \mathrm{C}$. Plasma carotenoids (including lycopene isomers) and vitamin $\mathrm{A}$ (retinol and retinyl palmitate) were extracted and analysed by Craft Technologies, Inc., using previously described procedures $^{(40,41)}$. In brief, plasma mixed with ethanol, water and butylated hydroxytoluene was extracted twice with hexane, concentrated under $\mathrm{N}_{2}$, and the extract was reconstituted in ethyl acetate and a mobile phase consisting of acetonitrile, dioxane, and methanol with ammonium acetate and triethylamine. The resolubilised extract was injected onto an isocratic HPLC system utilising the described mobile phase and was equipped with a Spherisorb ODS2 (C18) column with a guard column of the same stationary phase.

Prostate-specific antigen analysis. Venous blood was collected in $3 \mathrm{ml} \mathrm{K}_{3}$ EDTA tubes (Becton Dickinson) for PSA analysis. Blood samples were centrifuged, and plasma or serum was collected and immediately analysed for PSA concentrations using a competitive immunoassay with chemiluminescence (Bayer Diagnostics).

Prostate tissue carotenoid analysis. Prostate samples were immediately snap-frozen in liquid $\mathrm{N}_{2}$ and then stored at $-80^{\circ} \mathrm{C}$. Carotenoid extraction (approximately $0.05 \mathrm{~g}$ ) was conducted as described previously ${ }^{(11)}$. Prostate lycopene isomers were analysed as described previously ${ }^{(42)}$ with several modifications. The HPLC system consisted of a Hewlett-Packard 1050 (Santa Clara) solvent delivery system interfaced with a fourchannel CoulArray ${ }^{\mathrm{TM}}$ electrochemical detector (ESA) with potentials set between 380 and 560 in $60 \mathrm{mV}$ increments from channels 1 to 4 . Separations were carried out using an analytical YMC C30 column $(4.6 \times 150 \mathrm{~mm}, 5 \mu \mathrm{m})$ (Waters). Ammonium acetate (1.0 M, pH 4.6) was prepared with HPLCgrade water. Solvent A consisted of methanol-methyl tert-butyl ether-water-aqueous ammonium acetate (88:5:5:2), and solvent $\mathrm{B}$ consisted of methanol-methyl tert-butyl etheraqueous ammonium acetate (20:78:2). Separation of carotenoids and carotenoid isomers was performed at $1.0 \mathrm{ml} / \mathrm{min}$ using the following linear gradient: $0-5 \mathrm{~min}, 100 \%$ solvent A; 5-45 min, 15\% solvent A, $85 \%$ solvent B; 45-50 min, 100\% solvent B. Peak identification and quantification were determined with authentic standards for zeaxanthin, $\beta$-cryptoxanthin (Indofine), $\alpha$-carotene, $\beta$-carotene and lycopene (Sigma Chemical Company). Data collection and integration were performed using ESA CoulArray ${ }^{\mathrm{TM}}$ version 1.01 software and a data management system.

\section{Statistical analysis}

Results are expressed as means with their standard errors, unless otherwise noted. The study group sizes were selected 
based on a minimum six subjects needed to provide $80 \%$ power to detect a significant $(\alpha \leq 0.05) \quad 40 \%$ difference between final prostate lycopene concentrations, which was based on previous data ${ }^{(43)}$. Comparisons of changes in plasma lycopene concentration from baseline to post-intervention within each group were made using paired $t$ tests. ANOVA followed by Tukey's post hoc test was used to compare the effect of intervention type on the end-of-study plasma and prostate concentrations of vitamin A and carotenoids between the groups. The impact of each intervention on plasma carotenoid or vitamin A concentrations from baseline to the day of surgery was determined using repeatedmeasures ANOVA on subjects for whom both measurements were available. The correlations between dietary lycopene intake, plasma lycopene and prostate tissue lycopene concentrations were determined by Pearson's correlation. The impact of daily lycopene intake on end-of-study plasma lycopene concentration was determined using a linear regression model in which baseline plasma lycopene concentrations were included as a covariate, and no other adjustments were made. We used linear regression to determine the correlation between cumulative lycopene exposure and end-of-study prostate lycopene concentrations after controlling for baseline plasma lycopene and BMI. Differences in prostate total lycopene and isomer concentrations were determined in a subgroup of men for whom sufficient tissue samples were available ( $n$ 26) by ANOVA, followed by Tukey's post hoc test. Outliers were identified using Dixon's $Q$ test. All statistics were considered significant at $P<0.05$ and were conducted using SPSS Statistics 20 software (SPSS, Inc.).

\section{Results}

\section{Subject characteristics and compliance}

A total of thirty-six men provided informed consent for participation. Of these, two men underwent surgery earlier than originally planned and, therefore, did not complete the minimum intervention time of 2 weeks. After enrolling, a third man was diagnosed with a second malignancy during preoperative testing and was subsequently excluded, resulting in a final sample of thirty-three men (control $n 11$, soup $n 8$, sauce $n 7$ and juice $n 7$ ). Only one man was excluded from the tissue lycopene analysis because of a tissue lycopene concentration that was an outlier, probably due to an unexpected delay in surgery that prolonged the intervention period (total $64 \mathrm{~d}$ ). There were no significant plasma lycopene concentration outliers. There was one possible grade 2 gastrointestinal adverse event, but no other toxicities were reported. Moreover, laboratory values to assess the toxicity of the study interventions were all within normal limits at both the time of randomisation and the day of surgery. Serum PSA concentrations did not differ between the groups at either baseline or prostatectomy (pooled values: initial 5.8 (SEM 0.6$) \mathrm{ng} / \mathrm{ml}$ and final 5.6 (SEM 0.60$) \mathrm{ng} / \mathrm{ml}$, $P=0.53$ ). Other baseline anthropometric and biochemical measures and staging are summarised in Table 1.

Over the mean study duration $(23$ (SEM 1.7) d), men in the three tomato groups consumed $100 \%$ of the targeted dose
Table 1. Anthropometric, biochemical and pathological characteristics of the study participants

(Mean values with their standard errors; number of participants and percentages)

\begin{tabular}{|c|c|c|}
\hline & Mean & SE \\
\hline \multicolumn{3}{|l|}{ Baseline characteristics ${ }^{*}$} \\
\hline Age (years) & 59.7 & 1.0 \\
\hline Height $(\mathrm{cm})$ & 178 & 1.3 \\
\hline Weight (kg) & 95 & $2 \cdot 7$ \\
\hline $\operatorname{BMI}\left(\mathrm{kg} / \mathrm{m}^{2}\right)$ & 30 & 0.9 \\
\hline Intervention duration (d) & 23 & 1.7 \\
\hline IGF-1 (nmol/l) & 18 & 1.4 \\
\hline Testosterone (nmol//) & $12 \cdot 4$ & 0.4 \\
\hline PSA (ng/ml) & $5 \cdot 22$ & 0.56 \\
\hline Surgical pathology† & $n$ & $\%$ \\
\hline \multicolumn{3}{|l|}{ Pathological stage } \\
\hline pT2a & 6 & 19 \\
\hline pT2b & 5 & 16 \\
\hline pT2c & 12 & 39 \\
\hline рТЗа & 3 & 10 \\
\hline pT3b & 4 & 13 \\
\hline pT4 & 1 & 3 \\
\hline \multicolumn{3}{|l|}{ Gleason sum } \\
\hline 5 & 1 & 3 \\
\hline 6 & 10 & 32 \\
\hline 7 & 14 & 45 \\
\hline 8 & 2 & 6 \\
\hline 9 & 2 & 6 \\
\hline 10 & 2 & 6 \\
\hline
\end{tabular}

IGF-1, insulin-like growth factor 1; PSA, prostate-specific antigen.

${ }^{*} n 33$.

† $n$ 31. One man had a cystoprostatectomy (prostatectomy for the cure of non-prostatic malignancy), and one man had benign prostatic hypertrophy, so they were excluded from pathological staging and grading analyses.

on 500 of a total of 533 possible intervention days, representing $94 \%$ compliance with the daily dose. The mean lycopene consumption from the three assigned tomato intervention products was as follows: 26 (SEm $0 \cdot 8) \mathrm{mg} / \mathrm{d}$ from the juice; 22 (sem 1.3) $\mathrm{mg} / \mathrm{d}$ from the sauce; 31 (sем 0.9) $\mathrm{mg} / \mathrm{d}$ from the soup. A higher intake of lycopene from the soup can be attributed to the following: when prepared with an equal volume of milk or water, one can of soup yields $2 \frac{3}{4}$ cups, representing the higher end of the targeted intervention range; however, the subjects found this volume to be more convenient to consume than the lower end of the targeted intervention range (two cups prepared). Self-reported consumption of lycopene-containing foods other than those provided was minimal (mean $<1 \mathrm{mg}$ lycopene/d across the three tomato groups). Men in the control group consumed an average of 1.07 (SEM 0.59$) \mathrm{mg} / \mathrm{d}$ and only exceeded $5 \mathrm{mg}$ lycopene on $11(6 \%)$ of a total of $186 \mathrm{~d}$.

\section{Plasma lycopene and lycopene isomers}

Total plasma lycopene concentration significantly increased in men consuming each of the three tomato interventions $(P<0.05)$ and was non-significantly decreased in the control group (Table 2 ). The end-of-study plasma lycopene concentration in each of the three tomato groups was significantly higher than that of the control group (Fig. 1). The soup 
intervention led to significantly higher plasma lycopene concentrations than the juice intervention, while the impact of sauce consumption on plasma lycopene concentration was not significantly different from that of either juice or soup consumption (Table 2). In the tomato products provided for the intervention, $79-91 \%$ of lycopene was in the all-trans form $^{(36)}$. There were no significant between-group differences in the percentage of all-trans- or cis-lycopene in plasma at enrolment. Soup consumption led to a significantly higher percentage of plasma all-trans-lycopene and lower percentage of total plasma cis-lycopene compared with the isomer distributions at enrolment and with the control group; however, the percentage of cis-lycopene in the plasma of men consuming the tomato soup did not significantly differ from the other tomato intervention groups (Table 3).

\section{Other plasma carotenoids, retinol and retinyl palmitate}

The carotenoids lycopene, $\alpha$-carotene, $\beta$-carotene, lutein, zeaxanthin, $\alpha$-cryptoxanthin or $\beta$-cryptoxanthin were readily detectable in plasma. At the initiation of the study, none of the four intervention groups showed significant differences in baseline plasma concentrations of carotenoids, retinol or retinyl palmitate (Table 2). While the three tomato intervention groups had similar plasma concentrations of retinyl palmitate and retinol regardless of time or group, men in the control group had significantly lower mean plasma concentrations of both retinyl palmitate and retinol at the time of surgery than those in the juice intervention group $(P<0.05)$, though the difference was not significant in comparison with men in the other two tomato groups. At the time of surgery, men consuming the tomato juice had significantly higher plasma concentrations of $\alpha$-carotene than those consuming the control diet or the tomato sauce. Additionally, there was a significant increase in plasma $\beta$-carotene concentration from baseline to surgery in men consuming the tomato soup and significantly higher plasma $\beta$-carotene concentration at the time of surgery in men consuming the juice and soup compared with those consuming the sauce. We found no other significant withingroup (pre- $v$. post-intervention) or between-group differences in the plasma concentrations of $\alpha$-carotene, total $\beta$-carotene or cis- $\beta$-carotene, lutein, zeaxanthin, $\alpha$-cryptoxanthin, or $\beta$-cryptoxanthin (Table 2). Relative to baseline, the control group had significantly lower plasma concentrations of lutein, zeaxanthin, $\alpha$-cryptoxanthin, $\beta$-cryptoxanthin and cis- $\beta$-carotene after the $23 \mathrm{~d}$ intervention, perhaps reflecting a change in intake before surgery.

\section{Prostate lycopene and lycopene isomers}

Regardless of the dietary group, cis isomers were the major geometric configuration of lycopene in all prostate samples (79 (SEm 0.06)\%), with five cis isomer peaks and all-trans-lycopene being detectable, as reported previously ${ }^{(42)}$, in the prostates of men in the tomato product intervention groups (pooled, 19 (sem 0.01$) \%$ of all-trans) and the control group (25 (sem 0.03$) \%$ of all-trans) (Fig. 2; Table 4 ). Prostate lycopene concentrations were analysed in men who had a sufficient 

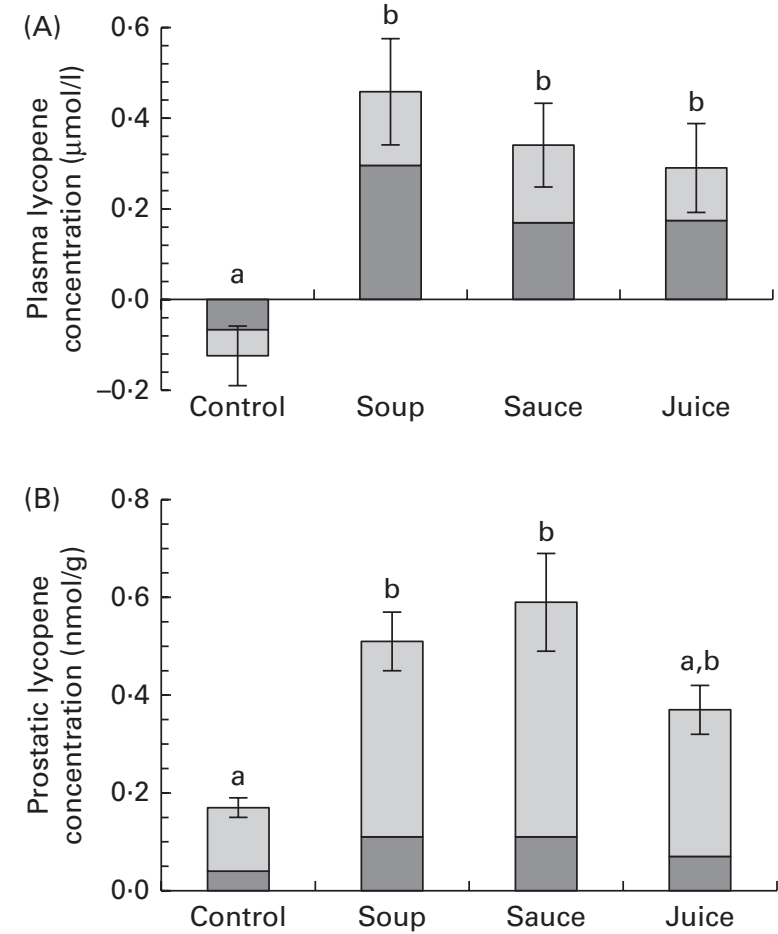

Fig. 1. Change in plasma lycopene $(n 7-11)(\mathrm{A})$ and final prostate lycopene $(n 6-7)$ (B) concentrations in control men or those consuming daily tomato soup, sauce or juice. Values are means, with their standard errors represented by vertical bars. ${ }^{a, b}$ Mean values with unlike letters were significantly different (ANOVA followed by Tukey's post hoc test). $\square$, Total cis-lycopene; $\square$, all-trans-lycopene.

quantity of tissue after pathological grading and staging ( $n$ 26; tomato intervention groups $n 19$ and control group $n$ 7). Prostate lycopene concentrations were 0.16 (SEM 0.02), 0.58 (SEM 0.06), $0.60($ SEM 0.10$)$ and $0.37($ SEM 0.07) nmol/g for the control, soup, sauce and juice groups, respectively. Compared with the control group, prostate lycopene concentrations were higher by $351 \%$ in men consuming the tomato soup ( $P=0.001), 362 \%$ in the tomato sauce group $(P=0.001)$ and $224 \%$ in the tomato juice group ( $P=0 \cdot 165$, NS). Men in the sauce and soup groups had higher prostate concentrations of all-trans-lycopene $(P<0.01$ for both), total cis-lycopene $(P<0.001$ for both) and 5-cis-lycopene $(P<0.01$ for both), compared with the control group (Fig. 1). Prostate total lycopene and lycopene isomer concentrations at the time of surgery did not differ significantly between the three tomato intervention groups.

\section{Other prostate carotenoids}

Prostate concentrations of non-lycopene carotenoids were analysed in the same subset of twenty-six samples. Prostate zeaxanthin $(0.11$ (SEM 0.02$) \mathrm{nmol} / \mathrm{g}), \beta$-cryptoxanthin $(0 \cdot 13$ (sem 0.03$) \mathrm{nmol} / \mathrm{g}), \alpha$-carotene $(0.06 \quad(\mathrm{sem} \quad 0.01) \mathrm{nmol} / \mathrm{g}$ ), $\beta$-carotene $(0.17$ (sem 0.02$) \mathrm{nmol} / \mathrm{g})$ and 9-cis- $\beta$-carotene (0.04 (SEM 0.01$) \mathrm{nmol} / \mathrm{g})$ concentrations were not significantly different between the intervention groups.

\section{Correlations between dietary lycopene intake, plasma lycopene and prostate lycopene}

We found a significant correlation between average daily lycopene intake over the intervention period (daily reported dietary intake plus intake from the intervention product $(\mathrm{mg} / \mathrm{d}))$ and final plasma lycopene concentration $(R \quad 0 \cdot 84$; $P<0.00001$ ) (Fig. 3). Additionally, plasma lycopene content at prostatectomy was modestly correlated with prostate lycopene content ( $r 0.60, P=0.001$; Fig. 3). In contrast, the relationship between dietary lycopene intake and final prostate lycopene concentration was significant, but noticeably lower, with the $r$ value being $0.41(P=0.046)$ and $0.40(P=0.04)$ for both average daily lycopene and cumulative lycopene intake, respectively (Fig. 3). This diet-prostate lycopene relationship was strongest among the six men in the soup group ( $r 0.86)$ compared with the other intervention groups. As expected, plasma lycopene concentrations at enrolment were poorly correlated with tissue lycopene concentrations after the dietary interventions $(r$ 0.29, $P=0 \cdot 11)$. Both plasma cis- and all-translycopene at the time of surgery were correlated with tissue cis- and all-trans-lycopene concentrations $(r$ 0.65, $P<0.001$ and $r 0.55, P<0.001$, respectively).

\section{Discussion}

The main objective of the present study was to quantitatively define how controlled consumption of processed tomato products in typical serving sizes could have an impact on blood

Table 3. Plasma lycopene geometric isomer distribution at enrolment and after dietary interventions with tomato food products

(Mean values with their standard errors)

\begin{tabular}{|c|c|c|c|c|c|c|c|c|}
\hline \multirow[b]{3}{*}{ Intervention groups } & \multicolumn{4}{|c|}{ Percentage of all-trans-lycopene } & \multicolumn{4}{|c|}{ Percentage of total cis-lycopene } \\
\hline & \multicolumn{2}{|c|}{ Baseline } & \multicolumn{2}{|c|}{ Surgery } & \multicolumn{2}{|c|}{ Baseline } & \multicolumn{2}{|c|}{ Surgery } \\
\hline & Mean & SEM & Mean & SEM & Mean & SEM & Mean & SEM \\
\hline Control (n 11) & 51 & 1 & $50^{\mathrm{a}}$ & 1 & 49 & 1 & $49^{a}$ & 1 \\
\hline Soup $(n 8)$ & 49 & 1 & $55^{b_{* *}}$ & 1 & 51 & 1 & $45^{\mathrm{b \star \star}}$ & 1 \\
\hline Sauce $(n 7)$ & 50 & 1 & $51^{a, b}$ & 1 & 50 & 1 & $49^{a, b}$ & 1 \\
\hline Juice $(n 8)$ & 51 & 1 & $55^{a, b}$ & 1 & 49 & 1 & $45^{a, b}$ & 1 \\
\hline
\end{tabular}

a,b Mean values with unlike superscript letters were significantly different $(P<0.05)$

${ }^{\star *}$ Mean value was significantly different from that at baseline $(P<0.01)$. 


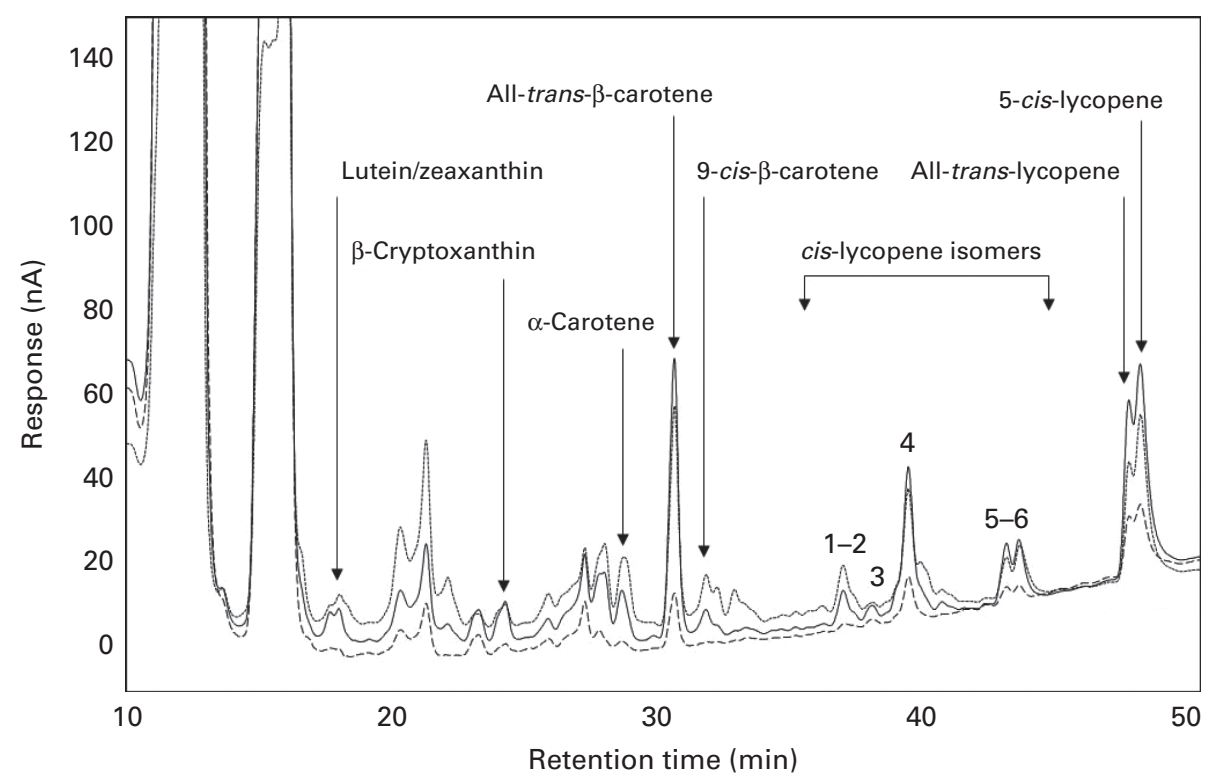

Fig. 2. Representative chromatogram of carotenoids detected in the prostate tissue, including multiple lycopene isomers (peaks 1-6), after the intake of tomato products. ...., $500 \mathrm{mV} ;-, 440 \mathrm{mV} ;-\cdot-, 380 \mathrm{mV}$.

and prostate lycopene concentrations. This essential information may help investigators to (1) interpret epidemiological studies that utilise FFQ and other indirect methods to estimate exposure to lycopene, (2) better design novel processed tomato products for clinical intervention studies, and (3) optimally design human studies investigating a potential causal relationship between tomato intake, lycopene, and prostate carcinogenesis or other disease outcomes. In the present study, subjects in the intervention arms successfully adhered to daily consumption of assigned tomato products in typical serving sizes for over 3 weeks before prostatectomy, while simultaneously following a diet restricted in all other tomato products and carefully documenting intake of both the study foods and other lycopene-containing foods. We found that tomato soup, sauce and juice can significantly increase plasma lycopene concentration, and that soup and sauce can particularly increase prostate lycopene concentrations. Critically, we found that, with precise quantification of lycopene intake, the correlations between dietary lycopene intake and plasma lycopene concentration are stronger than those reported by epidemiological studies that typically assess intake by FFQ. Nevertheless, correlations between dietary lycopene intake and prostate lycopene concentration or between plasma and prostate lycopene concentrations remain imperfect, indicating that many factors such as food processing, meal composition and host genetics/metabolism may contribute to individual variation in lycopene absorption and tissue distribution ${ }^{(33)}$.

\section{Impact of tomato products on plasma and prostate carotenoid concentrations}

We observed an average 65\% increase in plasma lycopene concentration from daily intake of standard servings of tomato soup, sauce and juice. In contrast, restriction of tomato consumption ( $<5 \mathrm{mg}$ lycopene/d) led to a $24 \%$ decrease in plasma lycopene concentrations. The decline in lycopene concentrations among the controls indicates that usual intake for a man in the present study was $>5 \mathrm{mg}$ lycopene/d. Plasma lycopene concentrations in the present study (mean 0.92 (SEm 0.06) $\mu \mathrm{mol} / \mathrm{l}$ across the intervention groups) are similar to those reported in our previous study after a sauce, soup or

Table 4. Prostate lycopene isomer concentrations and distributions after dietary interventions with tomato food products

(Mean values with their standard errors)

\begin{tabular}{|c|c|c|c|c|c|c|c|c|}
\hline \multirow[b]{3}{*}{ Intervention groups } & \multicolumn{8}{|c|}{ Prostate lycopene isomer concentration $(\mathrm{nmol} / \mathrm{g})$} \\
\hline & \multicolumn{3}{|c|}{ All-trans-lycopene } & \multicolumn{3}{|c|}{ Total cis-lycopene } & \multicolumn{2}{|c|}{$\begin{array}{c}\text { Total lycopene } \\
\text { (all-trans + total cis) }\end{array}$} \\
\hline & Mean & SEM & Percentage of total lycopene & Mean & SEM & Percentage of total lycopene & Mean & SEM \\
\hline Control (n 7) & $0.04^{\mathrm{a}}$ & 0.00 & 25 & $0.13^{a}$ & 0.02 & 75 & $0 \cdot 16^{a}$ & 0.02 \\
\hline Soup ( $(n 7)$ & $0.11^{\mathrm{b}}$ & 0.01 & 19 & $0.46^{\mathrm{b}}$ & 0.05 & 81 & $0.58^{\mathrm{b}}$ & 0.06 \\
\hline Sauce $(n 6)$ & $0.11^{\mathrm{b}}$ & 0.02 & 19 & $0.48^{\mathrm{b}}$ & 0.08 & 81 & $0.60^{\mathrm{b}}$ & 0.10 \\
\hline Juice $(n 6)$ & $0.07^{a, b}$ & 0.01 & 19 & $0.30^{a, b}$ & 0.05 & 81 & $0.37^{\mathrm{a}, \mathrm{b}}$ & 0.05 \\
\hline
\end{tabular}

${ }^{\mathrm{a}, \mathrm{b}}$ Mean values within a column with unlike superscript letters were significantly different $(P<0.01)$. 
(A)

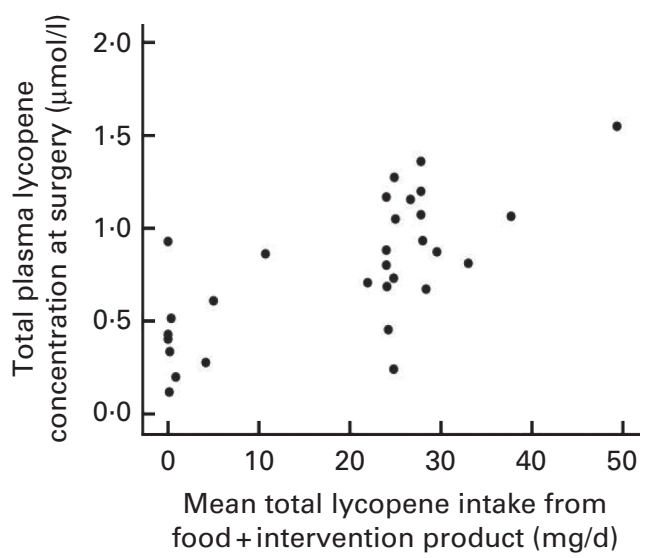

(C)

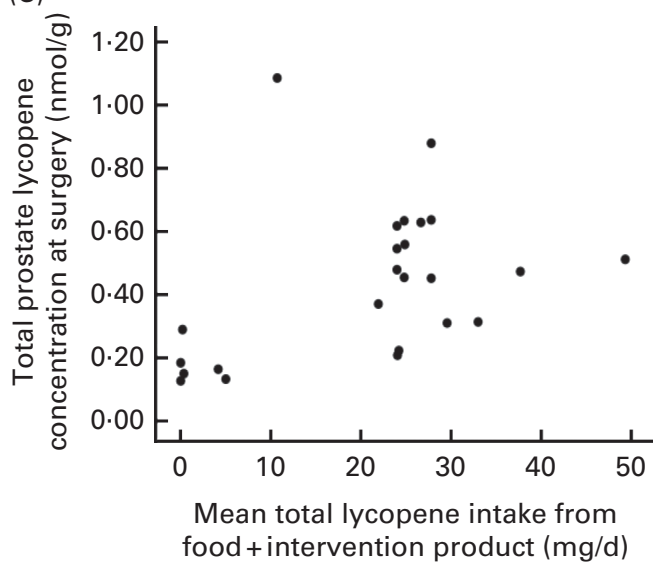

(B)

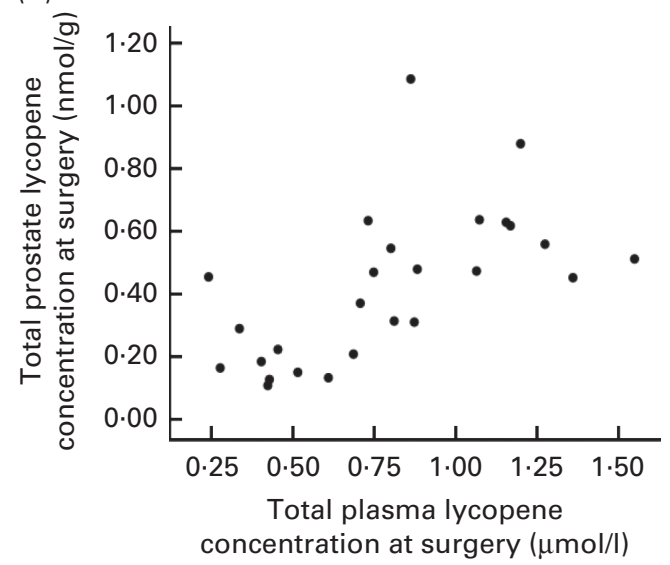

(D)

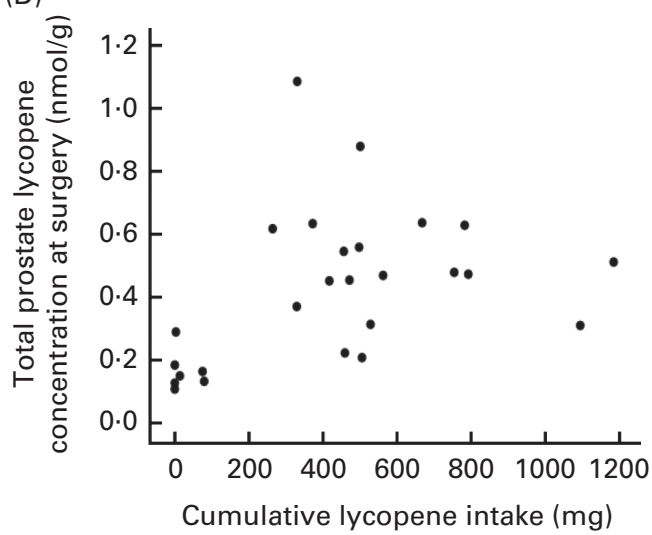

Fig. 3. Correlations between daily total dietary lycopene intake and post-intervention plasma lycopene concentration ( $n 30, r 0.84, P<0.00001)$ (A), plasma lycopene concentration after the washout period (baseline) and prostate lycopene concentration at the time of surgery $(n 26, r 0.60, P=0.001)(B)$, daily total dietary lycopene intake and prostate lycopene concentration at the time of surgery $(n 26, r 0.41, P=0.046)(C)$, and cumulative lycopene intake over the study intervention and prostate lycopene concentration at the time of surgery $(r 0.40, P=0.04)(D)$. Correlations in (A) were analysed by linear regression adjusted for baseline plasma lycopene concentrations, and correlations in $(B)$ and $(C)$ were determined by Pearson's correlation test. Data are representative of all samples of men consuming the tomato products $(n 21)$ and the control diet $(n 11)$ for a study duration of 23 (SEM 1.7) d.

juice intervention (mean $0.90 \mu \mathrm{mol} / \mathrm{l}$ across the intervention groups) ${ }^{(36)}$, and are slightly lower than those found in other tomato dietary intervention studies (mean $1 \cdot 3 \mu \mathrm{mol} / 1)^{(13,34,39)}$. Prostate lycopene concentrations are greater by 351, 362 and $224 \%$ in the soup, sauce and juice intervention groups, respectively, compared with the control group. In two earlier studies, daily consumption of tomato sauce for 3 weeks $30 \mathrm{mg}$ lycopene/d) has significantly increased prostate lycopene concentration by 2 - to 3 -fold ${ }^{(13,34)}$. Although we cannot compare final prostate lycopene concentrations with baseline prostate lycopene concentrations, we did observe that the final prostate lycopene concentrations (0.52 (SEM 0.05) nmol/g for all the three tomato groups) were generally similar to those previously reported for 'normal' prostate tissue in men with prostate cancer $(0.63(\mathrm{SEm} 0.09) \mathrm{nmol} / \mathrm{g})^{(11)}$ and were somewhat lower than those reported in two previous studies where men consumed tomato sauce daily for 3 weeks $(0.82$ and $1.09 \mathrm{nmol} / \mathrm{g})^{(13,34)}$. In one other pre-surgical study, men were randomised to either a tomato oleoresin capsule ( $n$ 15) providing $30 \mathrm{mg}$ lycopene/d or a placebo ( $n$ 11) for 3 weeks before prostatectomy. The end-of-study plasma lycopene levels were not significantly different between the groups $(0.438$ and $0.326 \mu \mathrm{mol} / 1$ in the intervention $v$. controls, respectively; $P=0 \cdot 15)$, but the end-of-study prostate lycopene concentrations were higher $(47 \%, P=0.02)$ in the intervention group $(0.99($ SEM 0.06$) \mathrm{nmol} / \mathrm{g})$ compared with the controls (0.67 (SEM 0.01) nmol/g); however, tissue was only procured from eight of the twenty-six men, which limits comparison with other studies ${ }^{(43)}$.

While the present study reports slightly lower prostate and plasma lycopene concentrations than previous studies ${ }^{(13,34)}$, this is likely related to restricting tomato food consumption outside the intervention (thus, lower total lycopene intake), unlike the other studies mentioned, in which tomato foods in addition to the intervention product were not controlled or measured. In general, soup and sauce resulted in greater increases in both plasma and prostate lycopene concentrations than did juice, which could be a result of the greater lipid content of these specific food types (soup lipid content ranges from 0.22 to $1.3 \%(\mathrm{w} / \mathrm{w})$ whether prepared with water or low-fat (2\%) milk; sauce contains $1.15 \%(\mathrm{w} / \mathrm{w})$, while juice has no appreciable lipid content $\left.(0 \%, \mathrm{w} / \mathrm{w})^{(32)}\right)$. 
Furthermore, co-consumed lipid from other components in the meal is probably an important factor facilitating lycopene absorption ${ }^{(33)}$. Juice may be more likely to be consumed alone and in between meals than soup and sauce, which are more likely to be consumed with a lipid-containing meal. Thus, lycopene absorption from juice may be improved if consumed with a lipid-containing meal or by adding lipid to the juice. Differences between lycopene bioavailability from processed tomato food products and resultant systemic lycopene exposure may be an important covariate when considering epidemiological data. Interestingly, in the Health Professionals Follow-Up Study, the association between tomato sauce and reduced risk of prostate cancer was significant, while the association between tomato juice and the risk of prostate cancer was not ${ }^{(1)}$.

Understanding the factors that influence prostate lycopene concentrations is relevant to elucidating a causal relationship between lycopene intake and prostate cancer prevention. Presently, there is no definitive work showing a dose-dependent relationship between prostate lycopene and subsequent prostate cancer development, although a recent small study has provided limited supportive evidence. Men ( $n$ 32) with high-grade prostatic intraepithelial neoplasia were enrolled to a single-arm, 6-month dietary tomato paste intervention (20-25 mg lycopene/d). An end-of-study biopsy found that men who were diagnosed with prostate cancer also had the lowest mean prostate lycopene concentrations (approximately $1.94 \mathrm{nmol} / \mathrm{g}$ ) compared with men whose end-of-study biopsy suggested prostatitis (approximately $3.52 \mathrm{nmol} / \mathrm{g}$ ) or highgrade prostatic intraepithelial neoplasia (approximately $3.61 \mathrm{nmol} / \mathrm{g})^{(44)}$. Other studies have examined surrogate endpoints after intervention ${ }^{(13,45,46)}$, but individual correlations between prostate lycopene concentrations and specific biomarkers were not reported.

A key point from the present study, in which lycopene intake from both the intervention product and the free-living diet was carefully documented, is that dietary lycopene intake and the resultant prostate lycopene concentration were only modestly correlated, with a correlation coefficient of 0.41 . Accordingly, it is clear that factors other than intake have an impact on tissue lycopene concentrations. Furthermore, this relatively modest relationship suggests that estimated intake from a FFQ, typically employed at a single point in time in many studies, is probably a modest, if not crude, overall predictor of lycopene exposure in prostate tissue, which may be critical to defining the biological impact. Therefore, the imprecision of dietary intake assessment and the multiple factors affecting absorption and metabolism within the framework of the complex epidemiology of prostate cancer may underlie the inability of many studies to detect a significant relationship between dietary tomato products and the risk of prostate cancer, if, indeed, there is a causal relationship ${ }^{(5)}$.

It is logical to hypothesise that epidemiological studies focusing on serum lycopene as a biomarker of lycopene exposure and the risk of prostate cancer is a more powerful approach. In the present study, we observed that plasma lycopene at the time of surgery is a better predictor of prostate lycopene $(r 0 \cdot 60, P=0 \cdot 001)$ than is estimated lycopene intake. However, a single plasma measure alone is insufficient to predict prostate content, as $40 \%$ of the variation remains unaccounted for in the present study. While we found a stronger relationship between plasma and prostate lycopene than did Bowen et al. ${ }^{(13)}$ ( $r$ 0.47, $P=0.01$ ), our findings were very similar to a previously reported correlation between plasma lycopene concentration and breast adipose tissue lycopene concentration in women with cancer and benign breast disease $\left(\begin{array}{ll}R & 0.61\end{array}\right)^{(47)}$. Other studies of plasma and tissue concentrations have focused on adipose tissue, with modest relationships existing primarily between abdominal

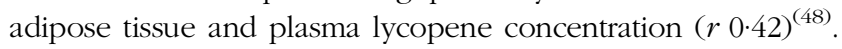
Consequently, the present findings suggest that plasma lycopene alone is a crude predictor of prostate lycopene exposure. Future clinical trials utilising a variety of dietary lycopene doses and resulting in a range of plasma and tissue concentrations and biological outcomes should be performed to improve the precision of a predictive model to describe the relationships between lycopene intake, plasma and prostate concentrations, and the risk of prostate cancer.

\section{Plasma and prostate concentrations of carotenoids}

Carotenoids share several of the same membrane transporters, and previous reports have suggested that some carotenoids may compete for absorption ${ }^{(49-52)}$. Because in vitro evidence suggests that lycopene can inhibit intestinal uptake of $\beta$-carotene ${ }^{(52)}$, the present study sought to determine whether incorporation of dietary sources of lycopene would incidentally depress plasma or prostate concentrations of other dietary carotenoids. In fact, tomato consumption was not associated with a decrease in plasma concentrations of any carotenoid or vitamin A; however, it was associated with an increase in plasma $\beta$-carotene concentrations after soup consumption (Table 2 ), perhaps due to dietary $\beta$-carotene contribution from the product. Furthermore, the dietary tomato interventions over 3 weeks did not have an impact on the concentrations of zeaxanthin, $\beta$-cryptoxanthin, $\alpha$-carotene, $\beta$-carotene or 9-cis- $\beta$-carotene in human prostate tissue.

\section{Impact of dietary interventions on isomer distribution in the plasma and prostate}

While the majority of dietary lycopene is typically in the all-trans geometric configuration ${ }^{(36)}$, tissue lycopene is present primarily as cis isomers ${ }^{(11)}$. Yet, we lack an understanding of this phenomenon and its relevance to biological outcomes. Some data suggest that cis-lycopene is a more efficient scavenger of peroxyl radicals ${ }^{(53)}$ and is the preferred substrate for $\beta$-carotene oxygenase 2 cleavage $^{(54)}$, a potential route of bioactivation and/or degradation. Dietary tomato consumption increases the proportion of all-trans-lycopene in prostate tissue and plasma ${ }^{(13)}$, but whether different processed tomato food products vary in their impact on prostate isomer distribution was previously unknown. In plasma, all-trans-lycopene accounted for between 50 and $55 \%$ of total lycopene. We found $19 \%$ of post-intervention prostate lycopene to be in the all-trans 
configuration, which is similar to that found in previous reports $^{(11,34)}$. Specifically, tomato soup, sauce and juice increased the amount of all-trans-lycopene in the prostate by 302, 303 and $77 \%$, respectively, compared with the control diet. Increases in prostate all-trans-lycopene concentration may reflect the recent all-trans-lycopene intake, supporting the concept that all-trans-lycopene is absorbed and then endogenously isomerised ${ }^{(36,55,56)}$. Nevertheless, the relative percentage of plasma all-trans-lycopene was slightly greater at both baseline and post-intervention (50 (SEM 1) and 54 (SEM 1) \%, respectively) than that previously reported in prostate cancer patients before and after a dietary tomato intervention (29 (SEM 6) and 32 (SEM 8)\%, respectively) ${ }^{(34)}$, suggesting that different tomato products could have different impacts on plasma lycopene isomer distributions. In general, these proportions coincide with the initially reported plasma all-trans-lycopene of $27-42 \%$ in prostate cancer patients ${ }^{(11)}$.

The limitations of the present study include the slight differences in the lycopene dose in each intervention arm, which was allowed to attain the goal of studying convenient serving sizes of tomato foods consumed in the marketplace. The strengths of the present study include excellent participant compliance, detailed records of non-intervention tomato intake, and analysis of human plasma and prostate lycopene concentrations following an intervention with relevant serving sizes of common tomato products.

\section{Conclusion}

In conclusion, we found that popular and commonly consumed tomato products increase plasma and prostate lycopene concentrations in men with prostate cancer when consumed in achievable daily serving sizes for 3 weeks. Nevertheless, even with precise quantification of lycopene consumption, dietary intake was only modestly correlated with plasma and prostate concentrations. Plasma lycopene at the time of surgery is a modest predictor of prostate lycopene. We demonstrated that the relationships between dietary intake of lycopene, blood and tissue concentrations, and biological responses are complex. Dietary and biological factors, including genetics, which influence blood and tissue lycopene concentrations, are not well understood, and more research is critical to clarify the relationship between lycopene and the risk of prostate cancer.

\section{Acknowledgements}

The authors thank Christina Simpson for proof reading and formatting the manuscript and Jessica Cooperstone for analysing the carotenoid profiles of the tomato products.

The present study was funded by the OSU Comprehensive Cancer Center (OSUCCC) (NIH/NCI P30 CA016058) and the OSU Center for Advanced Functional Foods and Entrepreneurship (CAFFRE). The prostate lycopene isomer analysis was conducted at the Nutrient and Phytochemical Analytic Shared Resource of the OSUCCC. Philanthropic support was provided by the James Cancer Hospital Bionutrition and Chemoprevention Fund and the Prostate Cancer Prevention
Fund, which have been supported by hundreds of donors over 15 years, including the Campbell Soup Company. The processed tomato products were donated by the Campbell Soup Company, so the Campbell Soup Company was apprised of the study design but had no role in the data analysis or in the writing of this article.

The authors' contributions are as follows: S. K. C., S. J. S., E. M. G. and C. W. H. designed the study; S. K. C., S. J. S., C. W. H., E. M. G., N. E. M. and K. M. R. formulated the research questions; S. K. C., M. C. G. and K. P. recruited the subjects; S. K. C., E. M. G. and C. W. H. carried out the clinical study; E. M. G., C. W. H. and K. M. R. analysed the samples; E. M. G., C. W. H., K. M. R., N. E. M., S. J. S. and S. K. C. analysed the data; C. W. H., E. M. G., N. E. M. and S. K. C. drafted the manuscript. All authors reviewed and approved the final manuscript.

Conflict of interest: S. K. C. was a paid consultant on the scientific policy advisory board for the Campbell Soup Company (2004), reviewing and supporting their letters to the Food and Drug Administration recommending that it was premature to request a health claim for lycopene or lycopene-containing foods. S. J. S. has served as a paid consultant for the Campbell Soup Company regarding food chemistry and composition of tomatoes and tomato products. The rest of the authors have no conflicts of interest.

\section{References}

1. Giovannucci E, Ascherio A, Rimm EB, et al. (1995) Intake of carotenoids and retinol in relation to risk of prostate cancer. J Natl Cancer Inst 87, 1767-1776.

2. Giovannucci E, Rimm EB, Liu Y, et al. (2002) A prospective study of tomato products, lycopene, and prostate cancer risk. J Natl Cancer Inst 94, 391-398.

3. Zu K, Mucci L, Rosner BA, et al. (2014) Dietary lycopene, angiogenesis, and prostate cancer: a prospective study in the prostate-specific antigen era. J Natl Cancer Inst 106, djt430.

4. Kristal AR, Arnold KB, Neuhouser ML, et al. (2010) Diet, supplement use, and prostate cancer risk: results from the prostate cancer prevention trial. Am J Epidemiol 172, 566-577.

5. World Cancer Research Fund (2014) Diet, Nutrition, Physical Activity and Prostate Cancer. Continuous Update Project. London: World Cancer Research Fund International.

6. Wei MY \& Giovannucci EL (2012) Lycopene, tomato products, and prostate cancer incidence: a review and reassessment in the PSA screening era. J Oncol 2012 271063.

7. Boileau TW, Liao Z, Kim S, et al. (2003) Prostate carcinogenesis in $N$-methyl- $N$-nitrosourea (NMU)-testosterone-treated rats fed tomato powder, lycopene, or energy-restricted diets. J Natl Cancer Inst 95, 1578-1586.

8. Konijeti R, Henning S, Moro A, et al. (2010) Chemoprevention of prostate cancer with lycopene in the TRAMP model. Prostate 70, 1547-1554.

9. Zuniga KE, Clinton SK, Erdman JW, et al. (2013) The interactions of dietary tomato powder and soy germ on prostate carcinogenesis in the TRAMP model. Cancer Preven Res $\mathbf{6}$, 548-557.

10. Canene-Adams K, Lindshield BL, Wang S, et al. (2007) Combinations of tomato and broccoli enhance antitumor activity 
in dunning r3327-h prostate adenocarcinomas. Cancer Res 67, 836-843.

11. Clinton SK, Emenhiser C, Schwartz SJ, et al. (1996) cis-trans Lycopene isomers, carotenoids, and retinol in the human prostate. Cancer Epidemiol Biomarkers Prev 5, 823-833.

12. Khachik F, Carvalho L, Bernstein PS, et al. (2002) Chemistry, distribution, and metabolism of tomato carotenoids and their impact on human health. Exp Biol Med (Maywood) 227, 845-851.

13. Bowen P, Chen L, Stacewicz-Sapuntzakis M, et al. (2002) Tomato sauce supplementation and prostate cancer: lycopene accumulation and modulation of biomarkers of carcinogenesis. Exp Biol Med (Maywood) 227, 886-893.

14. Rao AV, Fleshner N \& Agarwal S (1999) Serum and tissue lycopene and biomarkers of oxidation in prostate cancer patients: a case-control study. Nutr Cancer 33, 159-164.

15. Clinton SK (1999) The dietary antioxidant network and prostate carcinoma. Cancer 86, 1629-1631.

16. Kelkel M, Schumacher M, Dicato M, et al. (2011) Antioxidant and anti-proliferative properties of lycopene. Free Radic Res 45, 925-940.

17. Erdman JW Jr, Ford NA \& Lindshield BL (2009) Are the health attributes of lycopene related to its antioxidant function? Arch Biochem Biophys 483, 229-235.

18. Khan N, Afaq F \& Mukhtar H (2008) Cancer chemoprevention through dietary antioxidants: progress and promise. Antioxid Redox Signal 10, 475-510.

19. Liu X, Allen JD, Arnold JT, et al. (2008) Lycopene inhibits IGF-I signal transduction and growth in normal prostate epithelial cells by decreasing DHT-modulated IGF-I production in co-cultured reactive stromal cells. Carcinogenesis 29, 816-823.

20. Campbell JK, Stroud CK, Nakamura MT, et al. (2006) Serum testosterone is reduced following short-term phytofluene, lycopene, or tomato powder consumption in F344 rats. J Nutr 136, 2813-2819.

21. Siler U, Barella L, Spitzer V, et al. (2004) Lycopene and vitamin $\mathrm{E}$ interfere with autocrine/paracrine loops in the Dunning prostate cancer model. FASEB J 18, 1019-1021.

22. Wan L, Tan HL, Thomas-Ahner JM, et al. (2014) Dietary tomato and lycopene impact androgen signaling- and carcinogenesisrelated gene expression during early TRAMP prostate carcinogenesis. Cancer Prev Res (Phila) 7, 1228-1239.

23. Bureyko T, Hurdle H, Metcalfe JB, et al. (2009) Reduced growth and integrin expression of prostate cells cultured with lycopene, vitamin $\mathrm{E}$ and fish oil in vitro. Br J Nutr 101, 990-997.

24. Wertz K (2009) Lycopene effects contributing to prostate health. Nutr Cancer 61, 775-783.

25. Herzog A, Siler U, Spitzer V, et al. (2005) Lycopene reduced gene expression of steroid targets and inflammatory markers in normal rat prostate. FASEB J 19, 272-274.

26. McCarty MF (2004) Targeting multiple signaling pathways as a strategy for managing prostate cancer: multifocal signal modulation therapy. Integr Cancer Ther 3, 349-380.

27. Palozza P, Colangelo M, Simone R, et al. (2010) Lycopene induces cell growth inhibition by altering mevalonate pathway and Ras signaling in cancer cell lines. Carcinogenesis 31, 1813-1821.

28. Michaud DS, Giovannucci EL, Ascherio A, et al. (1998) Associations of plasma carotenoid concentrations and dietary intake of specific carotenoids in samples of two prospective cohort studies using a new carotenoid database. Cancer Epidemiol Biomarkers Prev 7, 283-290.

29. Hendrickson SJ, Willett WC, Rosner BA, et al. (2013) Food predictors of plasma carotenoids. Nutrients 5, 4051-4066.
30. Hodge AM, Simpson JA, Fridman M, et al. (2009) Evaluation of an FFQ for assessment of antioxidant intake using plasma biomarkers in an ethnically diverse population. Public Health Nutr 12, 2438-2447.

31. Thomson CA, Stendell-Hollis NR, Rock CL, et al. (2007) Plasma and dietary carotenoids are associated with reduced oxidative stress in women previously treated for breast cancer. Cancer Epidemiol Biomarkers Prev 16, 2008-2015.

32. U.S. Department of Agriculture Agricultural Research Service (2013) National Nutrient Database for Standard Reference Release 26. Beltsville, MD: National Agricultural Library.

33. Moran NE, Erdman JW Jr \& Clinton SK (2013) Complex interactions between dietary and genetic factors impact lycopene metabolism and distribution. Arch Biochem Biophys 539, $171-180$.

34. van Breemen RB, Xu X, Viana MA, et al. (2002) Liquid chromatography-mass spectrometry of cis- and all-translycopene in human serum and prostate tissue after dietary supplementation with tomato sauce. J Agric Food Chem 50, 2214-2219.

35. van Breemen RB, Sharifi R, Viana M, et al. (2011) Antioxidant effects of lycopene in African American men with prostate cancer or benign prostate hyperplasia: a randomized, controlled trial. Cancer Prev Res (Phila) 4, 711-718.

36. Allen CM, Schwartz SJ, Craft NE, et al. (2003) Changes in plasma and oral mucosal lycopene isomer concentrations in healthy adults consuming standard servings of processed tomato products. Nutr Cancer 47, 48-56.

37. USDA Economic Research Service (2013) Tomatoes and potatoes are the most commonly consumed vegetables. Economic Research Service, United States Department of Agriculture website.

38. Cooperstone JL, Ralston RA, Riedl KM, et al. (2015) Enhanced bioavailability of lycopene when consumed as cis-isomers from tangerine compared to red tomato juice, a randomized, cross-over clinical trial. Mol Nutr Food Res 59, 658-669.

39. Grainger EM, Schwartz SJ, Wang S, et al. (2008) A combination of tomato and soy products for men with recurring prostate cancer and rising prostate specific antigen. Nutr Cancer 60, 145-154.

40. Nomura AM, Stemmermann GN, Lee J, et al. (1997) Serum micronutrients and prostate cancer in Japanese Americans in Hawaii. Cancer Epidemiol Biomarkers Prev 6, 487-491.

41. Craft NE (2008) Laboratory Procedure Manual: A/E/Carotene Vitamin Profile in Serum NHANES 2003-2004. Washington, DC: CDC.

42. Ferruzzi MG, Nguyen ML, Sander LC, et al. (2001) Analysis of lycopene geometrical isomers in biological microsamples by liquid chromatography with coulometric array detection. J Chromatogr B Biomed Sci Appl 760, 289-299.

43. Kucuk O, Sarkar FH, Djuric Z, et al. (2002) Effects of lycopene supplementation in patients with localized prostate cancer. Exp Biol Med (Maywood) 227, 881-885.

44. Mariani S, Lionetto L, Cavallari M, et al. (2014) Low prostate concentration of lycopene is associated with development of prostate cancer in patients with high-grade prostatic intraepithelial neoplasia. Int J Mol Sci 15, 1433-1440.

45. Chen L, Stacewicz-Sapuntzakis M, Duncan C, et al. (2001) Oxidative DNA damage in prostate cancer patients consuming tomato sauce-based entrees as a whole-food intervention. J Natl Cancer Inst 93, 1872-1879.

46. Kucuk O, Sarkar FH, Sakr W, et al. (2001) Phase II randomized clinical trial of lycopene supplementation before radical prostatectomy. Cancer Epidemiol Biomarkers Prev 10, 861-868. 
47. Yeum KJ, Ahn SH, Rupp de Paiva SA, et al. (1998) Correlation between carotenoid concentrations in serum and normal breast adipose tissue of women with benign breast tumor or breast cancer. J Nutr 128, 1920-1926.

48. Chung HY, Ferreira AL, Epstein S, et al. (2009) Site-specific concentrations of carotenoids in adipose tissue: relations with dietary and serum carotenoid concentrations in healthy adults. Am J Clin Nutr 90, 533-539.

49. Reboul E \& Borel P (2011) Proteins involved in uptake, intracellular transport and basolateral secretion of fat-soluble vitamins and carotenoids by mammalian enterocytes. Prog Lip Res 50, 388-402.

50. Kostic D, White WS \& Olson JA (1995) Intestinal absorption, serum clearance, and interactions between lutein and $\beta$-carotene when administered to human adults in separate or combined oral doses. Am J Clin Nutr 62, 604-610.

51. van den Berg H \& van Vliet T (1998) Effect of simultaneous, single oral doses of $\beta$-carotene with lutein or lycopene on the $\beta$-carotene and retinyl ester responses in the triacylglycerol-rich lipoprotein fraction of men. Am J Clin Nutr 68, 82-89.

52. During A, Hussain MM, Morel DW, et al. (2002) Carotenoid uptake and secretion by CaCo- 2 cells: $\beta$-carotene isomer selectivity and carotenoid interactions. $J$ Lip Res 43, 1086-1095.

53. Muller L, Goupy P, Frohlich K, et al. (2011) Comparative study on antioxidant activity of lycopene ( $Z$ )-isomers in different assays. I Agric Food Chem 59, 4504-4511.

54. Hu KQ, Liu C, Ernst $\mathrm{H}$, et al. (2006) The biochemical characterization of ferret carotene-9',10'-monooxygenase catalyzing cleavage of carotenoids in vitro and in vivo. J Biol Chem 281, 19327-19338.

55. Ross AB, Vuong le T, Ruckle J, et al. (2011) Lycopene bioavailability and metabolism in humans: an accelerator mass spectrometry study. Am J Clin Nutr 93, 1263-1273.

56. Teodoro AJ, Perrone D, Martucci RB, et al. (2009) Lycopene isomerisation and storage in an in vitro model of murine hepatic stellate cells. Eur J Nutr 48, 261-268. 